\title{
Impacto del mercurio en los ecosistemas colombianos y las técnicas aplicables para su biorremediación
}

\author{
Claudia Patricia Pinzón', Carlos Andrés Fajardo Gomez²
}

\author{
1 Tecnólogo en Saneamiento Ambiental, Universidad Nacional Abierta y a Distancia. ${ }^{2}$ Magister en Ciencias, \\ Docente Escuela de Ciencias Agrícolas, Pecuarias y del Medio Ambiente, Universidad Nacional Abierta y a \\ Distancia - UNAD, Investigador del Grupo de Investigación Conservación, Bioprospección y Desarrollo \\ Sostenible (COBIDES-UNAD). \\ 1․ㅣ53pin174@unadvirtual.edu.co , 2 andres.fajardo@unad.edu.co
}

\begin{abstract}
Resumen
El trabajo de investigación está enfocado en una revisión bibliográfica del impacto que ha tenido el uso del mercurio en los ecosistemas colombianos, y tiene como objetivo analizar en el contexto colombiano las fuentes de contaminación, el manejo y la disposición final de este metal pesado así como las estrategias que se han implementado en el país para la recuperación de los ecosistemas afectados, con el fin de determinar las ventajas de las técnicas de biorremediación versus las técnicas fisicoquímicas, que en su mayoría requieren uso de aditivos químicos que pueden tener efectos secundarios y perjudiciales en el ecosistema tratado. La metodología a implementar en la investigación es una revisión bibliográfica del impacto causado por el mercurio en varios ecosistemas del país con la perdida de especies nativas de flora y fauna, y de la calidad del agua, aire y suelo del lugar. De otra parte, con la comparación de las técnicas fisicoquímicas con las técnicas de biorremediación se pretende establecer la prioridad de la biorremediación como la mejor alternativa para recuperar los ecosistemas afectados por mercurio debido al uso de microorganismos que se pueden integrar al medio sin afectar su composición natural y favoreciendo la recuperación de las características propias del ecosistema.
\end{abstract}

Palabras claves: Biorremediación, contaminación, ecosistema, metal pesado.

\section{Introducción}

El mercurio es un metal pesado con unas propiedades muy especiales que son apetecidas en la industria minera, farmacológica e industrial. Es el único metal que permanece líquido a temperatura ambiente y tiene una excelente relación con el oro permitiendo su separación y obtención del residuo explotado por los mineros (Rowlatt, Este metal puede llegar al medio ambiente de forma natural por medio de erupciones volcánicas y de manera antrópica mediante residuos industriales 0 mineros. Lamentablemente, sus múltiples propiedades y beneficios son opacados por las consecuencias que trae su uso para la salud humana y el medio ambiente. El mercurio se ha convertido en un veneno circundante con gran facilidad de bioacumulación y biomagnificación dentro de la cadena trófica, lo que aumenta sus efectos y el riesgo de intoxicación y contaminación por contacto con el mismo (Gaioli, Amoedo, y González, 2012).

Aunque su uso data de la época de los alquimistas, hasta hace pocos años se empezó a dar conocimiento e importancia de sus efectos adversos debido a la contaminación continua generada desde 1932 hasta 1968 cuando la industria de fertilizantes, petroquímicos y plásticos Chisso Corporation vertió residuos de mercurio en la Bahía de Minamata (Japón) lo que originó la contaminación del agua, formando un compuesto de metilmercurio que es la forma más toxica del mercurio. Esto trajo como consecuencia malformaciones fetales y trastornos de neurodesarrollo en los recién nacidos principalmente. En las personas adultas se presentaron problemas neurosensoriales y trastornos cognitivos, Gaioli et al., (2012).

De ahí partió el interés de minimizar el uso del mercurio remplazándolo con otros elementos de menor impacto y más amigables con el medio ambiente para evitar el 
deterioro de los ecosistemas, así como consecuencias negativas en la salud humana. Adicional al minimizar su uso, es necesario focalizar esfuerzos en remediar y recuperar aquellos ecosistemas que han sido deteriorados por el uso de este metal (Rowlatt, 2013).

\section{El mercurio como agente contaminante}

El mercurio es un metal pesado altamente tóxico que ha sido utilizado en el transcurso de la historia en diferentes actividades. Se puede liberar al medio ambiente en eventos naturales como son las erupciones volcánicas o por actividades antropogénicas como la minería, especialmente la explotación aurífera ya que el mercurio es el único metal que tiene reacción de amalgama con el oro, por tal motivo es utilizado en la extracción del mismo. A pesar de su gran utilidad poco a poco se ha ido disminuyendo su uso debido a los efectos adversos en el medio ambiente y en la salud de las personas (Rowlatt, 2013).

\section{Bioacumulación y transporte en la cadena trófica}

El mercurio es fácilmente bioacumulable y se presenta en las cadenas tróficas en dos formas: orgánicas e inorgánicas. Dentro de las especies inorgánicas están el metal mercurio, el óxido de mercurio, el catión mercurio y el catión mercurioso y dentro de las 25 formas orgánicas el dimetil mercurio, el metil mercurio y el fenil mercurio. La principal incorporación del mercurio a las cadenas tróficas es a partir del metal mercurio ya que es el más volátil y a temperatura ambiente se sublima incorporándose a la atmosfera en forma de vapor (Doadrio, 2004). En el medio acuático, el metil mercurio es el que genera una mayor interferencia en la cadena trófica debido a la facilidad que tiene para atravesar las membranas celulares y ser absorbido por el organismo afectando principalmente el sistema nervioso debido a su toxicidad. Por su gran capacidad de mantenerse en el ambiente, este compuesto se bioacumula y magnifica en la cadena trófica repetidamente desde el zooplancton hasta el depredador que encabeza la cadena (Kehrig et al., 2017).

Los ecosistemas acuáticos son de los más afectados por la minería, de esta forma, las comunidades humanas se ven directamente afectados al alimentarse de peces provenientes de estos ecosistemas. En una investigación realizada en la zona de playa de Riohacha se evaluó los niveles de concentración de diferentes metales pesados en donde se confirmó que se presencia se representaba niveles para efectos agudos en aguas marinas y estuarinas para preservación a nivel nacional (Doria \& Deluque, 2015).

\section{Efectos tóxicos del mercurio sobre la salud de los seres vivos}

El mercurio se considera como un contaminante de prioridad para la Unión Europea, en la Directiva del Marco del Agua, en el Convenio Ospar y el PNUMA. La Comisión Europea en 2005 adopto una estrategia comunitaria para reducir los niveles de mercurio en el medio ambiente y en la exposición humana. En estudios recientes se ha mejorado el conocimiento frente a las diferentes reacciones del $\mathrm{Hg}$ antropogénico, su ciclo biogeoquímico y las principales vías de transformación en el medio ambiente donde las 27 especies más afectadas son organismos acuáticos principalmente los que encabezan la cadena trófica como el tiburón (León y Peñuela, 2011). La toxicidad del mercurio varía de acuerdo a la forma presente en el ambiente (Organica o inorgánica). Llega al cuerpo humano por el consumo de alimentos principalmente pescados y mariscos o por desempeñar labores donde hay exposición al metal tales como la pesca o la minería. El mercurio afecta en gran medida el sistema digestivo, el sistema nervioso e inmunitario, los riñones, la piel, los pulmones y los ojos (Gaioli, Amoedo, y González, 2012).

\section{Uso del mercurio en la industria colombiana}

El mercurio ha sido utilizado en la industria colombiana en diversas actividades industriales, pero se destaca principalmente en la Minería Artesanal a Pequeña Escala (MAPE), donde su uso ha sido tradicional y extensivo por generaciones. La MAPE ha generado un alto impacto con los residuos de mercurio producto de la amalgamación para la obtención del oro y los cuales son vertidos a las fuentes hídricas o a la atmosfera sin ningún control ni criterio de cuidado ocupacional y ambiental (Pantoja y Pantoja, 2016). Al ser una actividad tan arraigada dentro de la economía nacional como base de sustento para muchas familias que habitan en zonas de violencia e ilegalidad y que están 
totalmente desprotegidas por el Estado sin tener otras oportunidades de ingreso, ha tenido una trascendencia dentro del contexto nacional llevando a Colombia a convertirse en uno de los mayores consumidores de mercurio a nivel mundial (Pantoja y Pantoja, 2016). De acuerdo a la base de datos UN-Comtrade, que es un banco de información que se alimenta de la base de datos International Financial Statistics (IFS), la información para un periodo comprendido entre 2007 y 2013 , evidencia que el total de las exportaciones de mercurio registradas hacia Colombia es de 79,6 t. sobresaliendo una exportación realizada en Bélgica de 2007 de 60 t que no aparece registrada como importación en Colombia (Ministerio de Minas y Energía-Unidad de Planeación Minero Energética y Universidad de Córdoba, 2014). Al revisar las exportaciones de mercurio hacia Colombia, se observa que los principales exportadores son: Alemania, Argentina, Perú, España, México, Suecia, Chile, Bélgica, Australia, Brasil, Canadá, Singapur y Suiza (Ministerio de Minas y Energía Unidad de Planeación Minero Energética y Universidad de Córdoba, 2014).

\section{Ecosistemas colombianos afectados por el uso del mercurio}

los ecosistemas relacionados a diversas actividades antrópicas, pueden llegar a sufrir serios impactos; cambios en el uso del suelo, reducción de la diversidad y sus hábitats, contaminación de los cuerpos de agua son consecuencia de las malas prácticas de explotación de recursos. Las propiedades resilientes de los ecosistemas se ven afectadas por dichas aliteraciones al igual que la capacidad natural de ofrecer los servicios ambientales primordiales para la estabilidad de la sociedad (Corredor, Fonseca y Páez, 2006)

En el departamento del Chocó, el río Condoto que es uno de los principales afluentes del río San Juan ha recibido vertimientos de mercurio provenientes de las explotaciones mineras, estimados entre 200 y $1000 \mathrm{~kg} / \mathrm{año}$ alcanzando niveles detectables directamente en el río y en algunas especies de peces. Es de consideración ya que adicional al daño ambiental, la salud de la población ribereña se ve afectada ya que el río es la fuente abastecedora del acueducto de Condoto lo que aumenta el riesgo de exposición de la población (Sánchez y Cañón, 2010).

En una zona cercana a Puerto Inírida (Guainía), entre 1998 y 1999 se realizó un estudio con población voluntaria que tuviera en común ser residentes de la zona, ejercer labores de minería aurífera y haber incluido en su dieta pescado y agua de la zona de estudio durante los 5 años previos. Se tomaron muestras de cabello y sangre encontrando concentraciones de 2,8 y $89,2 \mu \mathrm{g} / \mathrm{g}$ en cabello y de 6,9 y $168 \mu \mathrm{g} / \mathrm{l}$ en sangre evidenciando que solo el $4.6 \%$ de los individuos tenían concentraciones de mercurio por debajo de los límites máximos permisibles. Adicionalmente la población manifestó tener síntomas como pérdida de peso, cansancio físico, disminución de la memoria y la productividad, temblor e insomnio (Idrovo, et al., 2001).

En el municipio de San Marcos en el sur del departamento de Sucre se realizó un estudio exploratorio-descriptivo donde se seleccionaron 20 personas representativas de la población para tomarles muestras de cabello.Adicionalmente se tomaron muestras de arroz crudo y se evaluaron los niveles de mercurio total y metilmercurio mediante la técnica de espectrofotometría de absorción atómica por vapor frío obteniendo como resultados que el arroz que se comercializa de forma suelta (arroz blanco de San Marcos) fue el único que tuvo una mínima concentración de mercurio total de $0,021 \mu \mathrm{g} / \mathrm{g}$ y solo el $5 \%$ de la muestra poblacional excedió la dosis de referencia de ingestión de $\mathrm{Hg}$ $(0,1 \mu \mathrm{g} / \mathrm{kg}$ de peso corporal $/$ día establecido por la EPA).Sin embargo, es importante el monitoreo constante para prevenir la contaminación por arroz o consumo de otros alimentos (Argumedo, Consuegra, Vidal, y Marrugo, 2013).

Durante el 2016 se produjeron 42 toneladas de oro en Colombia de los cuales solamente el $12,4 \%$ del mineral se considera legal. El excedente del metal extraído proviene de los más de 300 municipios del país en los que se extrae el metal sin ningún control por parte del estado según cifras proporcionadas por Santiago Ángel presidente de la Asociación Colombiana de Minería (Semana Sostenible y Mongabay Latinoamérica, 2016).

No existen cifras actualizadas acerca de la entrada de mercurio a Colombia. Los últimos datos reportados son de Investigaciones contratadas por el Ministerio de Medio Ambiente en 2010 y 2012. La Universidad de Antioquia dice que en 2009 entraron al país 352 toneladas de mercurio y las 
Naciones Unidas dicen que en ese mismo año ingresaron 130 toneladas, dato que se acerca más al Informe presentado en 2013 por la Unidad de Planeación Minero Energética (UPME) que dice que se importaron 150 toneladas en ese año (Semana Sostenible y Mongabay Latinoamérica, 2016). El hecho de que no haya unanimidad en la información reportada por las diferentes entidades frente a la cantidad de mercurio que ingresa al país (a pesar de que se considera como una sustancia altamente tóxica y de efectos adversos para la salud y el medio ambiente) y que no se mantengan datos actualizados año a año, evidencia que no se están aplicando los controles en las actividades industriales y de explotación en las cuales este elemento es utilizado. Si no hay controles en la fuente de ingreso, se hace aún más difícil controlar los vertimientos y las emisiones que se hacen de los residuos generados por el uso del mercurio y así dar un seguimiento al aumento o disminución del consumo de este metal en el país.

Lo más preocupante de la importación de mercurio, es que una gran parte va a parar a los ríos del Norte del Cauca, departamento que produce apenas el $3,7 \%$ de oro y tiene el $4,1 \%$ de las minas a nivel nacional. Sin embargo, es el tercer departamento que más consume mercurio después de Antioquia y Bolívar. El mayor impacto se genera teniendo en cuenta que en los municipios de Buenos Aires y Suarez (Cauca) para la extracción de 1 gramo de oro se utilizan 14 gramos de mercurio. El metal ha generado un impacto significativo en este departamento contaminando las fuentes hídricas destinadas al consumo humano lo cual se evidencio en los muestreos realizados en el Rio Ovejas demostrando que hay concentraciones de mercurio que exceden entre 50 y 1000 veces los límites permitidos por las normas colombianas, lo cual afecta considerablemente el ecosistema acuático presentando mortandad de peces y la salud de los habitantes evidenciada en casos de malformaciones, problemas de riñón y daños cerebrales, entre otros (Semana Sostenible y Mongabay Latinoamérica, 2016).

En una valoración de los impactos ambientales ocasionados por la minería en el rio guabas en el Valle del Cauca se encontraron niveles de impactos severos y críticos asociados a diferentes actividades mineras, en donde la evaluación de la amalgamación usando mercurio evidenció impactos severos en flora y fauna al igual que los medios físicos aire, suelo y agua. El problema puede aumentar en magnitud con la implementación de procedimientos poco de responsables desde el punto de vista ambiental, en donde el uso de explosivos y el de grandes cantidades de agua puede llegar a causar daños irreversibles (Gamboa, 2015)

Otro de los casos más representativos en el país se dio en la bahía de Cartagena donde funciono la planta de soda caustica ALCO Ltda. Desde 1973 hasta 1977, donde el mercurio se utilizaba en el cambio del proceso electrolítico eliminando sus emisiones al medio acuático generando una alta concentración de metilmercurio fácilmente bioacumulable en el ecosistema (Invemar, 2012).

La región de La Mojana en Antioquia es reconocida por ser uno de las más biodiversos del país, pero así mismo uno de las más afectadas por la concentración de metales pesados, especialmente el mercurio. Las fuentes hídricas son las principales receptoras de los residuos generados por la explotación de carbón, ferroníquel y oro. En el municipio de la Mojana se encuentran los brazos de los ríos San Jorge, Cauca y Magdalena extendiéndose desde Ayapel hasta el pie de monte de la Sierra Nevada de Santa Marta. Al realizar el muestreo y posterior análisis en el Laboratorio del Grupo de Investigación en Gestión y modelación ambiental GAIA de la Universidad de Antioquia, se determinó que se superan los límites permisibles en muestras de arroz, sedimentos y cabello humano (Corantioquia y Gaia-Universidad de Antioquia, 2008).

En el Distrito Minero de San Martin de Loba (San Martin de Loba y Barranco de Loba-Mina Santa Cruz) ubicado al sur del departamento de Bolívar, la actividad minera ha generado focos de contaminación en los entables de amalgamación y en los sitios donde se realiza quema de amalgamas de oro superando los niveles máximos permisibles $\left(200 \mu \mathrm{g} / \mathrm{m}^{3}\right)$. Esta contaminación se dispersa a zonas no contaminadas afectando ecosistemas y trayendo efectos adversos a la salud humana y al medio ambiente (Olivero et al. 2014).

\section{Tratamiento y disposición final de los residuos de mercurio en Colombia}


Aunque aún las estadísticas y la información respecto al uso del mercurio en el país no son del todo completa y confiable, en Colombia se ha establecido legislación enfocada a la disminución del uso del mercurio y al tratamiento y disposición final adecuada de sus residuos.

Tabla 1. Normatividad Vigente para la Disposición final de Residuos de Mercurio en Colombia

\begin{tabular}{l|l}
\hline Resolución $\mathbf{1 5 9}$ de $\mathbf{2 0 1 5}$ & $\begin{array}{l}\text { Establece lineamientos que deben cumplir los Prestadores de Servicios de Salud de Bogotá D.C. para } \\
\text { la eliminación de productos y dispositivos con contenido de mercurio y la sustitución por alternativas } \\
\text { seguras y tecnológicamente no contaminantes (Secretaria Distrital de Salud, 2015). }\end{array}$ \\
\hline \multirow{2}{*}{ Resolución 1511 de $\mathbf{2 0 1 0}$} & $\begin{array}{l}\text { Por la cual se establecen los sistemas de recolección selectiva y Gestión Ambiental de Residuos de } \\
\text { Bombillas y se adoptan otras disposiciones (Ministerio de Ambiente, Vivienda y Desarrollo Territorial, } \\
\text { 2010). } \\
\text { El objetivo de aplicar esta resolución es que los comercializadores y productores de bombillas con } \\
\text { tecnología fluorescente compacta, haluros, fluorescente tubular, vapor de sodio o vapor de mercurio } \\
\text { están obligados a formular, presentar e implementar sistemas individuales o colectivos de recolección } \\
\text { selectiva y de gestión ambiental de los residuos de las bombillas usadas (Ministerio de Minas y Energía } \\
\text { - Unidad de Planeación Minero Energética y Universidad de Córdoba, 2014). }\end{array}$ \\
\hline Resolución $\mathbf{1 2 9 7}$ de $\mathbf{2 0 1 0}$ & $\begin{array}{l}\text { Por la cual se establecen los Sistemas de Recolección Selectiva y Gestión Ambiental de Residuos de } \\
\text { Pilas y/o Acumuladores y se adoptan otras disposiciones (Ministerio de Ambiente, Vivienda y Desarrollo } \\
\text { Territorial, 2010). }\end{array}$ \\
\hline Resolución $\mathbf{6 9 3}$ de $\mathbf{2 0 0 7}$ & $\begin{array}{l}\text { Por la cual se establecen criterios y requisitos que deben ser considerados para los Planes de Gestión } \\
\text { de Devolución de Productos Pos consumo de plaguicidas (Ministerio de Ambiente, Vivienda y Desarrollo } \\
\text { Territorial, 2007). }\end{array}$ \\
\hline Ley $\mathbf{1 6 5 8}$ de $\mathbf{2 0 1 3}$ & $\begin{array}{l}\text { Por medio de la cual se establecen disposiciones para la comercialización y el uso de mercurio en las } \\
\text { diferentes actividades industriales del país, se fijan requisitos e incentivos para su reducción y } \\
\text { eliminación y se dictan otras disposiciones (Agencia Nacional de Minería, 2013). }\end{array}$ \\
\hline
\end{tabular}

\section{Técnicas fisicoquímicas para recuperación de ecosistemas afectados por mercurio}

\section{Carbón activado granular}

Una de las técnicas fisicoquímicas de remoción de mercurio es el uso de CAG (carbón activado granular). En el municipio de Gachetá (Colombia), se realizó un experimento para evaluar la capacidad de remoción de este sólido para el tratamiento del mercurio contenido en las aguas procedentes del drenaje de la minería. Para la efectividad de este proceso influyen las características del solido (área superficial, tamaño del poro y capacidad de intercambio iónico). Así mismo, influye la geometría de la columna utilizada y sus condiciones (velocidad de flujo, altura de lecho del adsorbente y la naturaleza del drenaje), (Rojas, Guerrero, Vásquez, y Valencia, 2012). 


\section{Destilación del mercurio}

El proceso consiste en una carga continua del material a tratar en un tambor de acero que puede tener varias bocas de entrada dependiendo del residuo a tratar. Se logra la vaporización del mercurio calentando hasta $700^{\circ} \mathrm{C}$ (normalmente $500^{\circ} \mathrm{C}$ ). Posteriormente el mercurio pasa por un sistema de enfriamiento donde el mercurio se condensa sobre una camada de mercurio líquido. La recuperación y pureza del mercurio pueden alcanzar el $99.99 \%$ y los subproductos obtenidos son enfriados para su posterior manejo, (Geymonat, 2011).

\section{Desmercurización térmica}

El proceso se realiza en batch sin agitación y con una temperatura de operación aprox. de $500^{\circ} \mathrm{C}$ y con un vacío de $-300 \mathrm{~g} / \mathrm{Cm}^{2}$. El sistema consiste en bandejas dispuestas una sobre otra dentro de un recipiente calentadas por resistencias evaporando así el mercurio y extrayéndolo al vacío y luego se realiza el proceso de condensación, (Geymonat, 2011).

\section{Encapsulación del mercurio}

Existen dos formas de encapsular el mercurio: la micro encapsulación que consiste en mezclar los residuos con el material antes de solidificar y la macro encapsulación donde se vierte el material sobre una cantidad de residuos utilizando como material de encapsulamiento fosfato cerámico. El objetivo del encapsulamiento es inmovilizar el residuo para evitar el contacto con agentes lixiviantes como el agua, (Berrio, Beltran, Agudelo, y Cardona, 2012).

\section{Técnicas de biorremediación para ecosistemas afectados por mercurio}

En términos de servicios ecosistémicos, la calidad y regulación del agua son provistos por ecosistemas que poseen una variedad de funciones hidrológicas (Corredor, Fonseca y Páez, 2006). La explotación minera, sobre todo la informal, afecta la dinámica de estas funciones. Por tanto, es imprescindible la investigación en estrategias para mitigar la contaminación por mercurio proveniente de la minería.

Los servicios ecosistémicos relacionados con la regulación y la calidad del agua provienen de ecosistemas que proveen una gran variedad de funciones hidrológicas importantes para el bienestar humano

A pesar de que existen técnicas fisicoquímicas de remediación, en Colombia aún no se han establecido con propiedad y de forma estricta la implementación de estas para la recuperación de los ecosistemas afectados. La biorremediación se presenta como una gran alternativa, económica y de fácil aplicación teniendo en cuenta la baja accesibilidad en recursos para estos fines, (Ministerio de Ambiente y Desarrollo Sostenible, 2012).

La biorremediación es un proceso mediante el cual se utilizan organismos para la recuperación de las condiciones naturales de ecosistemas afectados por contaminantes. Se pueden utilizar bacterias (biorremediación), hongos (micorremediación) o plantas (fitorremediación). La biorremediación consiste principalmente en la estimulación de microorganismos nativos para que actúen degradando el contaminante a compuestos menos tóxicos en un proceso de mineralización que conlleva la formación de biomasa y dióxido de carbono. En algunos ecosistemas la degradación se da en el ambiente mediante la atenuación natural o biodegradación intrínseca. Sin embargo, cuando la degradación se da lentamente, se requiere la aplicación de la bioestimulación identificando el factor limitante del ambiente para suministrarlo al medio y así favorecer la acción de los microorganismos, (Benítez, 2017).

\section{Atenuación natural}

Es un método que consiste en reducir las concentraciones de un contaminante en el ambiente mediante procesos biológicos propios del ecosistema (la biodegradación es uno de los procesos principales de la atenuación natural en el suelo donde los microorganismos actúan para cambiar la 
química del mismo), reacciones químicas como la transformación abiótica, el intercambio iónico y la complejación o por medio de procesos físicos como dispersión, dilución, difusión, volatilización, advección y sorción/desorción (Corona \& Iturbe, 2005).

\section{Bioestimulación}

En este proceso se estimula la actividad natural de los microorganismos en el suelo por medio de la circulación de soluciones con nutrientes y oxigeno u otro aceptor de electrones (Gómez et al., 2009).

\section{Bioaumentación}

La bioaumentación es la adición de microorganismos nativos, externos o genéticamente modificados al medio debido a la baja densidad de los microorganismos nativos o a la falta de capacidad metabólica para la degradación (Benítez, 2017).

La bioaumentación en un suelo contaminado se puede determinar caracterizando las poblaciones de microorganismos y evaluando cuáles de ellos son aptos para colonizar y degradar los contaminantes (Gómez et al., 2009).

\section{Biosorción}

La biosorción también se presenta como una alternativa viable para la remoción del mercurio del ambiente. La biosorción consiste en una alternativa tecnológica utilizando procesos de separación con biomateriales como algas marinas, o desechos provenientes de la producción industrial, de procesos biológicos o de la agricultura. Algunos residuos agro industriales han demostrado características adsorbentes, en diferentes tipos de efluentes industriales. Se ha usado residuos agrícolas como la cascarilla de arroz y la corteza de coco para la remoción de moléculas contaminantes.

En este proceso la calidad del material adsorbente se considera de acuerdo a la capacidad que tiene de atraer y retener el metal en una forma inmovilizada (Borja, Garcia, Yipmantin, Guzmán y Maldonado, 2015).

En cuanto a los metales pesados, la biosorción se da por medio de microorganismos utilizando biomasa viva o muerta para retirar o inmovilizar iones metálicos de un medio sólido o líquido a través de mecanismos físicos y químicos como la adsorción y el intercambio iónico. La biosorción involucra una fase sólida o biosorbente (membranas o paredes celulares) y una fase líquida o solvente (casi siempre es agua) en la cual están las especies metálicas a ser bioadsorbidas, (Mejía, 2006).

\section{Degradación enzimática}

Es una aplicación de la biotecnología que consiste en la producción de enzimas en bacterias modificadas genéticamente. Estas enzimas se emplean en el sitio contaminado con el fin de degradar las sustancias nocivas, (Di Paola y Vicién, s.f).

\section{Remediación microbiana}

Este proceso consiste en la aplicación en el sitio contaminado de microorganismos nativos o importados de otros ecosistemas para ser inoculados. Se evalúan las condiciones y capacidades del microrganismo y acorde a eso se estimula el medio con nutrientes como nitrógeno y fosforo para acelerar el proceso, (Di Paola y Vicién, s.f).

\section{Fitorremediación}

La fitorremediación es una aplicación natural in situ o ex situ que permite el uso de plantas o de microorganismos asociados a ellas para absorber, estabilizar, acumular o volatilizar contaminantes como metales pesados, metales radiactivos, compuestos orgánicos o hidrocarburos. La planta suele absorber los contaminantes en la raíz. Las tecnologías de fitorremediación se basan principalmente 
en los mecanismos fisiológicos básicos como: transpiración, fotosíntesis, metabolismo y nutrición, (Delgadillo, González, Prieto, Villagómez, y Acevedo, 2011).

\section{Ventajas de la biorremediación versus las técnicas fisicoquímicas}

Al realizar la comparación de los métodos fisicoquímicos y las técnicas de biorremediación para la remoción de mercurio se evidencia que la biorremediación trae más beneficios al medio ambiente ya que el tratamiento se puede realizar in situ con microorganismos nativos del ecosistema que pueden contribuir a la remoción del contaminante mediante la bioestimulación o la bioaumentación (Amaringo y Hormaza, 2013)

Al ser una técnica natural, la biorremediación no deja residuos perjudiciales para el medio ambiente como si sucede con las técnicas fisicoquímicas que pueden dejar residuos de los químicos utilizados en el proceso.

Por otro lado, al ser un proceso in situ la biorremediación es beneficiosa al permitir el ahorro en costos de transporte del contaminante o de maquinaria e infraestructura necesaria para su remoción.

Los procesos biológicos representan un avance hacía tecnologías más limpias siempre y cuando se superen los percances medio ambientales que puedan afectar el proceso y disminuir la conversión o mineralización de contaminantes, (Dussán, Vives, Sarria, y Sánchez, 2009).

La desventaja que se evidencia frente al tratamiento de los desechos de mercurio principalmente en los países de ALC (Acuerdo de Libre Comercio), es la falta de una normativa específica para la gestión de estos residuos y la limitación en cuanto a tecnología para el tratamiento y descontaminación. Por otro lado, los costos influyen en la adquisición de las tecnologías adecuadas y en la adecuación de los vertederos para evitar depositarlos con otros residuos, (Santana et al., 2014).

\section{Conclusiones}

- Al evaluar el impacto generado por el uso del mercurio en la Industria Nacional y en actividades de explotación como la minería frente al tratamiento que se da a sus residuos se evidencia una gran falencia ya que no existen procesos regulados para la disposición final de los mismos. La legislación colombiana es muy deficiente frente al tema aunque esta situación se deriva de una problemática mundial debido a que no se había considerado la peligrosidad del mercurio, sus efectos adversos en el medio ambiente y en la salud humana y la importancia de manipular adecuadamente sus residuos hasta que se estableció el acuerdo de Minamata que tiene como objetivo priorizar la atención sobre el mercurio como un contaminante de fácil expansión y bioacumulación en el ambiente y del cual se derivan situaciones de riesgo.

- Se hace difícil suprimir el uso del mercurio principalmente en comunidades que le han dado un uso tradicional y que lo utilizan a diario en procesos que son vitales para su fuente de sustento desconociendo las consecuencias inmediatas y a futuro por una continua exposición. Aunque en algunas comunidades los síntomas por contaminación con mercurio son evidentes, aun así, los habitantes no han descontinuado su uso y siguen vertiendo residuos de mercurio a las fuentes hídricas, la atmosfera y el suelo.

- Las técnicas fisicoquímicas pueden ser útiles para la recuperación del mercurio ya sea para almacenamiento o un posterior uso, pero no son eficientes en la eliminación de este contaminante. Por tal motivo, no se pueden considerar como técnicas de remediación 100 $\%$ eficientes.

- En Colombia se han desarrollado proyectos investigativos basados en procesos de biorremediación, con el fin de evaluar la recuperación de ecosistemas afectados por la contaminación con mercurio y así encontrar estrategias aplicables que sean económicas, 
eficientes y amigables con el medio ambiente. En estos procesos se han dado resultados interesantes basados en la remoción microbiana, el lombricompostaje y la micorremediación.

- La falta y unanimidad de información actualizada acerca del ingreso, uso y disposición final del mercurio en cada una de las actividades industriales y de explotación en las cuales es utilizado hace que el control sobre los residuos generados sea deficiente. Sumado a esto, parte de la industria minera opera sin licencias lo que implica que no hay exigencia en planes de manejo ambiental ni de mitigación de impactos incluyendo los derivados por el uso del mercurio trayendo consecuencias al medio ambiente y la salud humana.

- Otro aspecto relevante frente a la disposición inadecuada de los residuos derivados del uso del mercurio es la falta de información y capacitación a las personas que se involucran directamente con este metal y que no saben cómo protegerse de la exposición directa y como disponer los residuos sin afectar las fuentes hídricas, el suelo, la atmosfera o su propia salud por el no uso de elementos de protección.

- Adicional al tema de los residuos sin disposición final adecuada, se suma el alto costo de las tecnologías necesarias para realizar los tratamientos, motivo por el cual se ha retrasado la implementación de estrategias de recuperación del metal por cuestión económica. La adecuación de sitios para el tratamiento de estos residuos también es un asunto de gran influencia por la inversión que requiere y por lo que implica el minimizar al máximo los sub residuos que se puedan generar de los procesos.

- Ante la falta de recursos económicos para la implementación de altas tecnologías, la alternativa más viable para la recuperación de ecosistemas afectados es la biorremediación que se da como resultado de un proceso natural derivado de la adaptación de diversas especies a ciertos contaminantes. La biorremediación permite que los organismos endémicos o previamente adecuados al ecosistema realicen la remoción sin dejar efectos secundarios que puedan llegar a ser más perjudiciales que el suceso inicial.

- Con la investigación y estrategia adecuada se pueden determinar las especies idóneas para la recuperación de un ecosistema contaminado teniendo en cuenta la capacidad de metabolización y eliminación de la sustancia y el tiempo requerido para la realización del proceso.

\section{Literatura citada}

Agencia Nacional de Mineria. (2013). Agencia Nacional de Mineria. Ley No. 1658 (Julio 15 de 2013). Recuperado de: https://www.anm.gov.co/sites/default/files/ley 1658 2013.pdf

Amaringo Villa, F. (2013). Determinación del punto de carga cero y punto isoeléctrico de dos residuos agrícolas y su aplicación en la remoción de colorantes. Revista de Investigación Agraria y Ambiental, 4(2), 27-36. doi:https://doi.org/10.22490/21456453.982

Argumedo, M., Consuegra, A., Vidal, J., \& Marrugo, J. (2013). Exposición a mercurio en habitantes del municipio de San Marcos (Departamento de Sucre) debida a la ingesta de arroz (Oryza Sativa) contaminado. Revista de Salud Pública. Recuperado de: https://www.scielosp.org/article/rsap/2013.v15n6/903-915/

Benitez, Y. (2017). Estado del arte en metodos biotecnólogicos potenciales a ser empleados para el tratamiento de aguas residuales contaminadas con mercurio, proveniente de la minería aurifera (Tesis de pregrado). Universidad Católica de Manizales, Manizales. Recuperado de:

http://repositorio.ucm.edu.co:8080/jspui/bitstream/handle/10839/1785/Yeison\%20Javier\%2 OBenitez\%20Caicedo.pdf? sequence=1\&isAllowed $=y$ 
Berrio, L., Beltrán, O., Agudelo, E., \& Cardona, S. (2012). Sistemas De Tratamiento Para Residuos Líquidos Generados En Laboratorios De Análisis Químico. Gestión y Ambiente, 15(3), 113124. Recuperado de: https://revistas.unal.edu.co/index.php/gestion/article/view/36285/43153

Borja, N., Garcia, V., Yipmantin, A., Guzmán, E., \& Maldonado, H. (2015). Estudio de la cinética de biosorción en plomo (II) en Alga Ascophyllum Nodosum. Rev Soc Quím Perú. 81(3).

Corantioquia y Gaia-Universidad de Antioquia. (2008). Niveles de mercurio en sedimento, agua y tejido vivo "buchón, arroz, peces y cabello" en los humedales de La Mojana Sucreña.

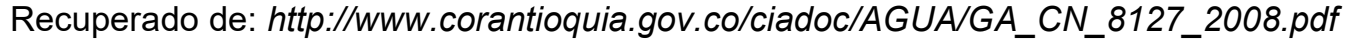

Corona, L., \& Iturbe, R. (2005). Atenuación natural en suelos contaminados con hidrocarburos. Redalyc, 119-126. http://dx.doi.org/10.22201/fi.25940732e.2005.06n2.008

Corredor Camargo, E., Fonseca Carreño, J., \& Páez Barón, E. (2012). Los servicios ecosistémicos de regulación: tendencias e impacto en el bienestar humano. Revista de Investigación Agraria y Ambiental, 3(1), 77-83. doi:https://doi.org/10.22490/21456453.936

Di Paola, M., \& Vicién, C. (s.f.). Biorremediación: vinculaciones entre investigación, desarrollo y legislación. Recuperado de:

https://www.researchgate.net/publication/262935161_Biorremediacion_vinculaciones_entre _investigacion_desarrollo_y_legislacion

Delgadillo, A., González, C., Prieto, F., Villagómez, J., \& Acevedo, O. (2011). Fitorremediación: una alternativa para eliminar la contaminación. Tropical an subtropical agroecosystems.

Recuperado: $h$ ttp://www.scielo.org.mx/scielo.php?pid=S187004622011000200002\&script=sci_arttext\#t3

Doria Argumedo, C., \& Deluque Viloria, H. (2015). Niveles y distribución de metales pesados en el agua de la zona de playa de Riohacha, La Guajira, Colombia. Revista de Investigación Agraria y Ambiental, 6(1), 123 - 131. doi:https://doi.org/10.22490/21456453.1268

Dussán, J., Vives, M., Sarria, V., \& Sánchez, O. (2009). Aproximaciones biológicas y fisicoquímicas en el tratamiento de contaminantes: un resumén del aporte de la Universidad de los Andes. Revista de Ingeniería. Recuperado de: https://ojsrevistaing.uniandes.edu.co/ojs/index.php/revista/article/view/233/231

Doadrio, A. (2004). Ecotoxicología y acción toxicológica del mercurio. Real Academia Nacional de Farmacia. Recuperado de: $h t t p: / / w w w . a n a l e s r a n f . c o m / i n d e x . p h p / a r a n f / a r t i c l e / v i e w / 254 / 283$

Gaioli, M., Amoedo, D., \& González, D. (2012). Impacto del mercurio sobre la salud humana y el ambiente. Archivos argentinos de pediatría. Recuperado de: http://www.scielo.org.ar/scielo.php?script=sci_arttext\&pid=S0325-00752012000300017

Gamboa, D. (2015). Valoración de impactos ecológicos por minería de oro en río Guabas, Valle del Cauca, Colombia. Riaa, 6(2), 243-254. Recuperado de: https://dialnet.unirioja.es/servlet/articulo?codigo $=5628792$

Geymonat, E. (2011). Manejo Racional de Productos con mercurio. Recuperado de: http://www.ccbasilea-crestocolmo.org.uy/wp-content/uploads/2010/11/Tratamientot\%C3\%A9rmico-para-la-recuperaci\%C3\%B3n-de-mercurio-Diciembre-2011.pdf

Gómez, W., Gaviria, J., \& Cardona, S. (2009). Evaluación de la bioestimulación frente a la atenuación natural y la bioaumentación en un suelo contaminado con una mezcla de gasolina-diesel. Dyna 76 (160), 83-93. Recuperado de: https://revistas.unal.edu.co/index.php/dyna/article/view/13471/14378 
Idrovo, Á., Manotas, L., Villamil, G., Ortíz, J., Silva, E., Romero, S., \& Azcárate, C. (2001). Niveles de mercurio y percepción del riesgo en una población minera aurifera del Guainía (Orinoquía Colombiana). Biómedica. Recuperado de: http://www.scielo.org.pe/scielo.php?pid=S1728$59172010000400016 \&$ script=sci_arttext\&tIng=en

Invemar. (2012). Informe del estado de los ambientes y recursos marino y costeros en Colombia año 2011 . Recuperado de: Boletin de Investigaciones Marinas y Costeras INVEMAR: http://www.invemar.org.co/redcostera1/invemar/docs/IER_2011.pdf

Kehrig, H., Baptista, G., Beneditto, A., Almeida, M., Rezende, C., Siciliano, S., . . Moreira, I. (2017). Biomagnificación de mercurio en la cadena trófica del Delfín Moteado del Atlántico (Stenella frontalis), usando el isótopo estable de nitrógeno como marcador ecológico. Revista de Biología Marina y oceanografía, 233-244. Recuperado de: https://scielo.conicyt.cl/pdf/revbiolmar/v52n2/art04.pdf

León, D., \& Peñuela, G. (2011). Trascendencia del metilmercurio en el ambiente, la alimentación y la salud humana. Producción + Limpia. Recuperado de: http://repository.lasallista.edu.co:8080/ojs/index.php/pl/article/view/135/69

Mejía, G. (2006). Aproximación teórica a la biosorción de metales pesados por medio de microorganismos. Revista CES Medicina Veterinaria y Zootecnia. Recuperado de: http://www.redalyc.org/html/3214/321428096010/

Ministerio de Minas y Energia-Unidad de Planeación Minero Energetica y Universidad de Cordoba. (2014). Estudio de la cadena del mercurio en Colombia con enfásis en la actividad minera de oro. Recuperado de: https://www.mesadedialogopermanente.org/wpcontent/uploads/2015/07/Cadena_Mercurio-Tomo-I-Prod-1-2-3-y-4.pdf

Ministerio de Ambiente, Vivienda y Desarrollo Territorial. (2010). Alcaldía Bogotá. Recuperado de: http://www.alcaldiabogota.gov.co/sisjur/normas/Norma1.jsp?i=40019

Ministerio de Ambiente, Vivienda y Desarrollo Territorial. (2007). IGAC. Recuperado de: http://www2.igac.gov.co/igac_web/normograma_files/Resolucion\%20693\%20de\%202007.p $d f$

Ministerio de Ambiente, Vivienda y Desarrollo Territorial. (2010). Recuperado de: http://www.minambiente.gov.co/images/AsuntosambientalesySectorialyUrbana/pdf/Program a_posconsumo_existente/RESOLUCION_1511_BOMBILLAS.pdf

Ministerio de Minas y Energía-Unidad de Planeación Minero Energética y Universidad de Córdoba. (2014). Estudio de la cadena del mercurio en Colombia con énfasis en la actividad minera de oro. Recuperado de: https://www.mesadedialogopermanente.org/wpcontent/uploads/2015/07/Cadena_Mercurio-Tomo-I-Prod-1-2-3-y-4.pdf

Olivero, J., Young, F., \& Caballero, K. (2014). Contaminación por mercurio en aire del Distrito Minero de San Martín de Loba en el Departamento de Bolívar, Colombia. Revista Internacional de Contaminación Ambiental. Recuperado de:: http://www.scielo.org.mx/scielo.php?pid=S018849992014000100001\&script=sci_arttext\&tIng=en

Pantoja, F., \& Pantoja, S. (2016). Problemas y desafíos de la minería de oro artesanal y en pequeña escala en Colombia. Revista de la facultad de ciencias ecónomicas: Investigación y reflexión. Recuperado de: http://www.scielo.org.co/pdf/rfce/v24n2/v24n2a09.pdf

Rojas, H., Guerrero, D., Vásquez, O., \& Valencia, J. (2012). Aplicación del módelo de Bohart y Adams en la remoción de mercurio de drenajes de minería por adsorción con carbón activado. Información tecnológica. Recuperado de: https://scielo.conicyt.cl/scielo.php?pid=S0718-07642012000300004\&script=sci_arttext 
Rowlatt, J. (2013). El mercurio, un metal bello pero fatal. BBC Mundo. Recuperado de: http://www.bbc.com/mundo/noticias/2013/12/131207_mercurio_contaminacion_peligros_or o_az_finde

Sánchez, D., \& Cañón, J. (2010). Análisis documental del efecto de vertimiento domésticos y mineros en la calidad del agua del río Condoto(Chocó, Colombia). Redalyc. Recuperado de: $h$ ttp://www.redalyc.org/html/1694/169419996009/

Santana, V., Medina, G., \& Torre, A. (2014). El Convenio de Minamata sobre el mercurio y su implementación en la región de América Latina y El Caribe. Centro Coordinador Convenio de Basilea. Recuperado de: http://www.ccbasilea-crestocolmo.org.uy/wpcontent/uploads/2014/05/Informe_Convenio-Minamata.pdf

Semana Sostenible y Mongabay Latinoamérica. (2016). Veneno en la Sangre. Recuperado de: http://sostenibilidad.semana.com/medio-ambiente/multimedia/mercurio-en-colombiaveneno-en-la-sangre/37266

Secretaria Distrital de Salud. (2015). Alcaldía Bogotá. Recuperado de: http://www.alcaldiabogota.gov.co/sisjur/normas/Norma1.jsp?i=61279 